\title{
IMPACT OF HYDROCHLOROTHIAZIDE ON THE STABILITY OF TWO PERINDOPRIL SALTS. EVALUATION OF THE INTERACTION WITH HPLC AND ESI LC/MS METHODS
}

\author{
ANNA JUSZCZAK ${ }^{1}$, WOJCIECH SZCZOŁKO², MARTYNA PIESZAK', \\ JUDYTA CIELECKA-PIONTEK ${ }^{1}$ and BEATA JADWIGA STANISZ ${ }^{1 *}$
}

\author{
${ }^{1}$ Department of Pharmaceutical Chemistry, Poznań University of Medical Sciences, \\ Grunwaldzka 6, Poznań, Poland \\ ${ }^{2}$ Department of Chemical Technology of Drugs, Poznań University of Medical Sciences, \\ Grunwaldzka 6, Poznań, Poland
}

\begin{abstract}
Perindopril (PER) belongs to the group of the angiotensin-converting enzyme inhibitors and is widely prescribed antihypertensive drug. It can be used in monotherapy or in combination therapy for example with hydrochlorothiazide (HTH). As on the market there is no pharmaceutical formulation containing both drugs and in literature has not been reported any work about effect of HTH on PER degradation process, the primary objective of this study was the assessment of stability of two salts of perindopril - tert-butylamine (PERt) and arginine (PERa) in a mixtures model with HTH in different relative humidities and constant temperature. The objective of the study was establishing the mechanisms of drug decomposition in the presence of HTH. Results were achieved using the high-performance liquid chromatography (RP-HPLC). The degradation rate constants for mixtures and pure substances were calculated. Decomposition products have been analyzed by ESI LC/MS and the decomposition mechanism for each salt has been proposed. The degradation of PERt in the presence of HTH took place according to autocatalytic reaction kinetic mechanism, described mathematically by ProutTompkins equation, and the decomposition process leads to hydrolysis. HTH in the model mixture with PERa generates a first-order kinetic model of the decomposition reaction, and there are two main products of decomposition: product of hydrolysis and diketopiperazine. Our study showed that HTH has statistically significant positive impact on both salts. It can be suggested that PERt or PERa and HTH can be formulated together, hence there is no negative interaction between the drugs.
\end{abstract}

Keywords: perindopril tert-butylamine, perindopril arginine, hydrochlorothiazide, drug stability, interaction

Perindopril (PER) - (2S,3aS,7aS)-1-[(2S)-2[[(2S)-1-ethoxy-1-oxopentan-2-yl]amino]propanoyl]-2,3,3a,4,5,6,7,7a-octahydroindole-2-carboxylic acid-belongs to the group of angiotensinconverting enzyme inhibitors (ACEI) and has been extensively described as effective in the treatment of hypertension, as drugs delaying progression in diabetic nephropathy, reducing mortality in left ventricular dysfunction and congestive heart failure (13 ). PER can be used in monotherapy or in combination therapy for example with thiazides (TZ), which are predominantly represented by the hydrochlorothiazide (HTH) (4).

Therapy of hypertension with fixed-dose combinations

Hypertension, as the most common cardiovascular disease among the world, belongs to the main factors increasing the risk of life-threatening complications and leads to about $30 \%$ deaths worldwide (5). Therefore hypotensive therapy, as so often used by the society, should intend to be effective as much as possible and prevent side effects. Fixed-dose combination is a modern and efficient model of hypotensive therapy, thereby differing mechanisms of action allows to strengthen the pharmacological effect, reduce adverse events and improve patient's comfort (6).

Combination therapy with an angiotensin converting enzyme inhibitor and a diuretic

Research has shown that combination therapy with ACEIs and diuretic is more effective than monotherapy and there is a possibility of starting the treatment with the lowest dose of drugs. PER is working very well in patients at increased risk or

\footnotetext{
* Corresponding author: e-mail: bstanisz@ump.edu.pl
} 
heart diseases, diabetic patients, after cerebrovascular accidents or in the elderly (7). The most commonly used salts of PER in the pharmaceutical market are PER tert-butylamine (PERt) and PER arginine (PERa) - Figure 1. Clinical studies have shown that PER and HTH work synergistically. They have different compatibility mechanisms. Fixed dose combination with PERt or PERa and HTH is better tolerated and more effective in the treatment of hypertension than monotherapy. Moreover, there is no difference in the main pharmacokinetic parameters in monotherapy and combination therapy, adverse events have been reported rarely and were minor (8). Higher doses of hydrochlorothiazide (more than $50 \mathrm{mg}$ ) do not grow hypotensive effect but only the side effects of the drug. A reduction of dosage helps to minimize the adverse metabolic effect and increases medication tolerability (9).

\section{Stability of drug}

To obtain a new formulation of the drug, it is necessary to investigate the stability of the molecules. Stability levels and hence long shelf-lives are required for drug's formulations. Drug stability assessment is used to approve efficacy before the expiry date, and only high quality means safety and valid therapeutic results. High temperature and humidity are the most important factors of drugs degradation, thus for avoiding toxicity or lack of treatment effect, stability and influence of high temperature and humidity should be monitored as often as possible (10-12). From a chemical point of view, it was reported that HTH in substance, when is stored at room temperature, is stable for five years (10). In previous studies authors have proved that PERt like others ACEIs is not stable drug when is exposed to high temperature and relative humidity, therefore also the influence of HTH in new potential drug formulation should be investigated (13-22).

Until now there were no available reports and no existing data evaluating kinetic parameters of degradation of PERt or PERa in the presence of HTH in a model mixture. PERt and PERa in solid state were investigated, whereas on the pharmaceutical market are available in solid state, in form of tablets, the same as HTH. The present study aims at the stability of PERa and PERt in model mixtures with HTH in terms of the influence of the air relative humidity (RH) and temperature (T) on its decomposition process. New information about kinetic model of degradation that this study provides can induce investigations of novel pharmaceutical formulations.

\section{EXPERIMENTAL}

\section{Materials}

PER tert-butylamine (PERt) was supplied by Bachem, (serial number L-2780-004-020). PER arginine (PERa) was supplied by Apotex Pharmachem India (serial number PEPFA00912), Hydrochlorothiazide (HTH) was purchased from SigmaAldrich (Germany). Methanol and acetonitrile were HPLC grade (Merck, Germany). All other chemicals were of analytical reagent grade. Potassium dihydrogen phosphate $\left(\mathrm{KH}_{2} \mathrm{PO}_{4}, \mathrm{M}=136.09 \mathrm{~g} / \mathrm{mol}\right)$ and sodium chloride were obtained from $\mathrm{POCH}$ (Gliwice, Poland).

\section{Methods and equipment \\ Liquid chromatography}

The chromatographic separation was achieved using LiChroCART ${ }^{\circledast}$ 250-4 HPLC-Cradridge column, LiChrospher ${ }^{\circledR} 100$ RP-18 (5 mm) (Merck, Germany), which worked at ambient temperature and the HPLC with a system consisting of a Rheodyne 7120, $20 \mathrm{~mL}$ fixed-loop injector, an LC 3-UV spectrophotometric detector (Pye Unicam, England, UK), an L-6000A pump (Merck-Hitachi, Darmstadt, Germany). The detector was set at 215 $\mathrm{nm}$ and peak areas were transmitted with Chromed software (Medson, Poland).

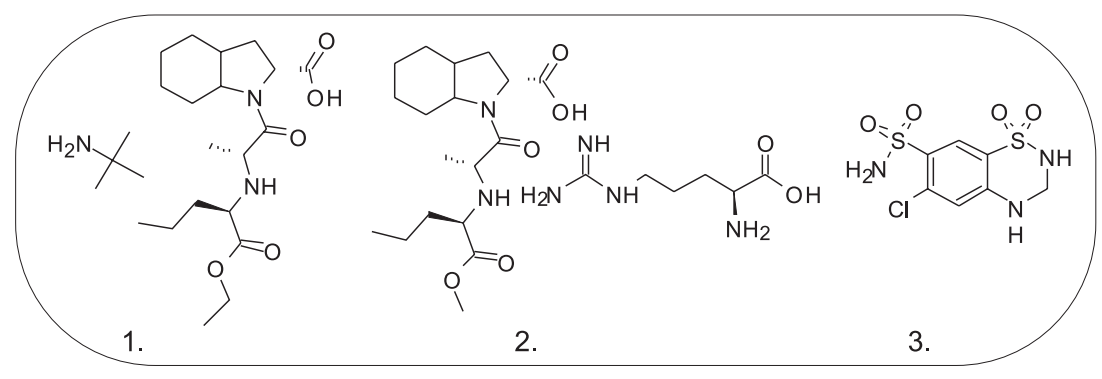

Figure 1. Structural PERt (1), PERa (2) and HTH (3) 


\section{Conditions of kinetic studies}

Appropriate relative humidity levels and temperature were obtained in heat chamber KBF 240 (Binder). Samples for the kinetic study were consisted of $15.00 \mathrm{mg}$ accurately weighed model mixture powder placed in an amber glass, uncapped vials. The samples were exposed to the conditions of kinetic study $\mathrm{T}=333 \mathrm{~K}$ and various relative humidity levels $(76.4 \%, 66.5 \%, 50.9 \%)$.

\section{Electrospray ionization-mass spectrometry system}

Electrospray ionization (ESI), was recorded by the Advanced Chemical Equipment and Instrumentation Facility at the Faculty of Chemistry, Adam Mickiewicz University in Poznań.

\section{Solutions \\ The mobile phase for liquid chromatography}

The applied mobile phase consisted of acetonitrile - phosphate buffer $(0.001 \mathrm{~mol} / \mathrm{L}$, adjusted to pH 2.0 with orto-phosphoric acid) $(70: 30, \mathrm{v} / \mathrm{v})$. It had been filtered through a filter $0.22 \mathrm{mM}$ and degassed by ultrasound prior to use. Aqueous phosphate buffer was prepared by dissolving $68.1 \mathrm{mg}$ of $\mathrm{KH}_{2} \mathrm{PO}_{4}$ in $450 \mathrm{~mL}$ of bidistilled water. It was adjusted to $\mathrm{pH} 2.0$ using phosphoric (V) acid (85\%) and completed to $500 \mathrm{~mL}$ with bidistilled water.

\section{Stock solutions}

Stock solutions of PERa and PERt were prepared by dissolving $10.00 \mathrm{mg}$ of each compound in $25.0 \mathrm{~mL}$ of methanol. The stock solutions were stored at $280 \mathrm{~K}$ with constant temperature monitoring, protected from light, for 10 days with no evidence of decomposition. The series of standard solutions ranging from 0.06 to $0.4 \mathrm{mg} / \mathrm{mL}$ were obtained by the appropriate dilutions of the stock solutions with methanol.

\section{Procedures}

Procedures for validation of HPLC method for analysis

Calibration procedure

Calibration curves of PERt and PERa for the HPLC analysis were determined by the linear regression analysis. The series of methanolic standard of each compound at 10 concentrations $(0.06$, $0.08,0.12,0.16,0.20,0.24,0.28,0.32,0.36,0.4$ $\mathrm{mg} / \mathrm{mL}$ ) were prepared. All the solutions were chromatographed immediately after preparation. HPLC analysis was conducted with $20 \mathrm{~mL}$ injections for each standard solution (for three times). The chromatograms were evaluated on basis of drug ratios of peak areas, and they were plotted against correspon- ding concentrations to assess linearity. The limits of detection (LOD) is described by the equation: LOD $=3.3 \mathrm{SD} / \mathrm{a}$, limit of quantification (LOQ) were evaluated on basis of the equation: LOQ $=10 \mathrm{SD} / \mathrm{a}$; where SD stands for the standard deviation and a stands for the slope of the calibration curve.

\section{Precision}

The precision of a method is expressed by the relative standard deviation of replicate measurements. Ten replicate determinations of two different concentrations of investigated drugs (low, $\mathrm{c}=0.06$ $\mathrm{mg} / \mathrm{mL}$; high, $\mathrm{c}=0.4 \mathrm{mg} / \mathrm{mL}$ ) were conducted on the same day in order to evaluate the repeatability.

\section{Selectivity}

The selectivity of the HPLC method was adequate and chromatograms demonstrate the separation of PERa or PERt, their degradation products, and $\mathrm{HTH}$, achieved during the chromatographic process.

\section{Model mixtures}

Perindopril tert-butylamine - hydrochlorothiazide model mixture (I) - MM I

A synthetic model mixture of PERt and HTH was prepared by grinding both components manually in ceramic mortar for $30 \mathrm{~min}$ at room temperature until homogeneous. The ratio $8: 12.5$ (w/w) was selected, while PERt is used in therapy in doses 4 $\mathrm{mg}$ or $8 \mathrm{mg}$, HTH is used in doses $12.5 \mathrm{mg}$ or $25 \mathrm{mg}$.

Perindopril arginine - hydrochlorothiazide model mixture (II) - MM II

A synthetic model mixture of PERa and HTH was prepared by grinding both components manually in ceramic mortar for $30 \mathrm{~min}$ at room temperature until homogeneous. The ratio $10: 12.5(\mathrm{w} / \mathrm{w})$ was selected, while PERa is used in therapy in doses 5 $\mathrm{mg}$ or $10 \mathrm{mg}, \mathrm{HTH}$ is used in doses $12.5 \mathrm{mg}$ or 25 mg (medications available on the market with HTH itself or HTH with ACEI contain $12.5 \mathrm{mg}$ or $25 \mathrm{mg}$ of $\mathrm{HTH})$.

\section{Samples preparation for HPLC analysis}

After the definite time intervals, the vials were removed from the heat chamber, cooled to room temperature and dissolved in methanol. Each sample $(15.00 \mathrm{mg}$ ) of model mixtures after the process of incubation was precisely and quantitatively transferred to a volumetric flask and subsequently filled up to $25.0 \mathrm{~mL}$ volume with methanol and mixed (procedure - preparation of model mixtures). Samples were subjected $(20 \mathrm{~mL})$ to HPLC analysis in order to measure quantitatively the content of 
PERt or PERa in the presence of its degradation product.

The decrease in PERt or PERa concentration of analyzed samples after incubation were calculated with respect to the stock solution.

\section{RESULTS AND DISCUSSION}

\section{Results of validation}

The selected RP-HPLC method was validated in order to confirm its applicability for this study. Precision, accuracy, selectivity, linearity, limits of quantitation and detection were evaluated according to ICH guidelines and literature $(23,24)$.

The HPLC method with UV detection applied in this study was found to be suitable for the determination of PERa and PERt in the presence of HTH under isothermal kinetic test conditions. (25-28) The method selectivity towards PERa and PERt in the presence of HTH and their degradation products (Dec 1, Dec 2) was proved. Chromatograms are con- sisted of regular, sharp and well-separated peaks characterized by retention times: $\sim 2 \mathrm{~min}, 3.5 \mathrm{~min}$; $4.3 \mathrm{~min} 5.5 \mathrm{~min}$, which matched: HTH, product/ products of decomposition and PERa or PERt (Fig. 2 ). The results of the validation procedure are summarized in Table 1. After detailed analysis, the method was qualified for further kinetic studies.

\section{Results of kinetic studies}

The kinetic studies of the investigated PERa and PERt were performed by means of the validated RP-HPLC method. The kinetic studies of the MM I and MM II were carried under conditions of relative humidity $(76.4 \%, 66.5 \%, 50.9 \%)$ and constant temperature $(333 \mathrm{~K})$. The samples were taken from the heat chamber, dissolved, analyzed and then the results were calculated and compared with the results of previous kinetic studies (carried out in the same conditions) of PERa and PERt in pure. The kinetic mechanism of degradation and proper kinetic equations are demonstrated in Table 2 and Table 3.

Table 1. The results of the validation of RP-HPLC method.

\begin{tabular}{|l|c|c|}
\hline \multicolumn{1}{|c|}{ Parameters } & PERa & PERt \\
\hline Linearity range [mg/mL] & $0.06-0.4$ & $0.06-0.4$ \\
Regression equation $y=$ ax & $7.77 \pm 0.27$ & $2.70 \pm 0.09$ \\
Slope a \pm a & 0.118 & 0.043 \\
Standard deviation of the slope (Sa) & 0.043 & 0.015 \\
Standard deviation (SD) & 0.999 & 0.998 \\
Correlation coefficient (r) & 0.018 & 0.018 \\
Limits of detection (LOD) & 0.055 & 0.055 \\
Limit of quantification (LOQ) & $1.48 ; 1.98$ & $1.55 ; 1.99$ \\
Precision (RSD, low; high [\%]) & $99.54 \pm 0.3$ & $99.86 \pm 0.5$ \\
Recovery [\%] & & \\
\hline
\end{tabular}

Table 2. The calculated decomposition rate constans of PERt in pure and PERt in model mixture with HTH $(\mathrm{MM} \mathrm{I})$; T = 333 K.

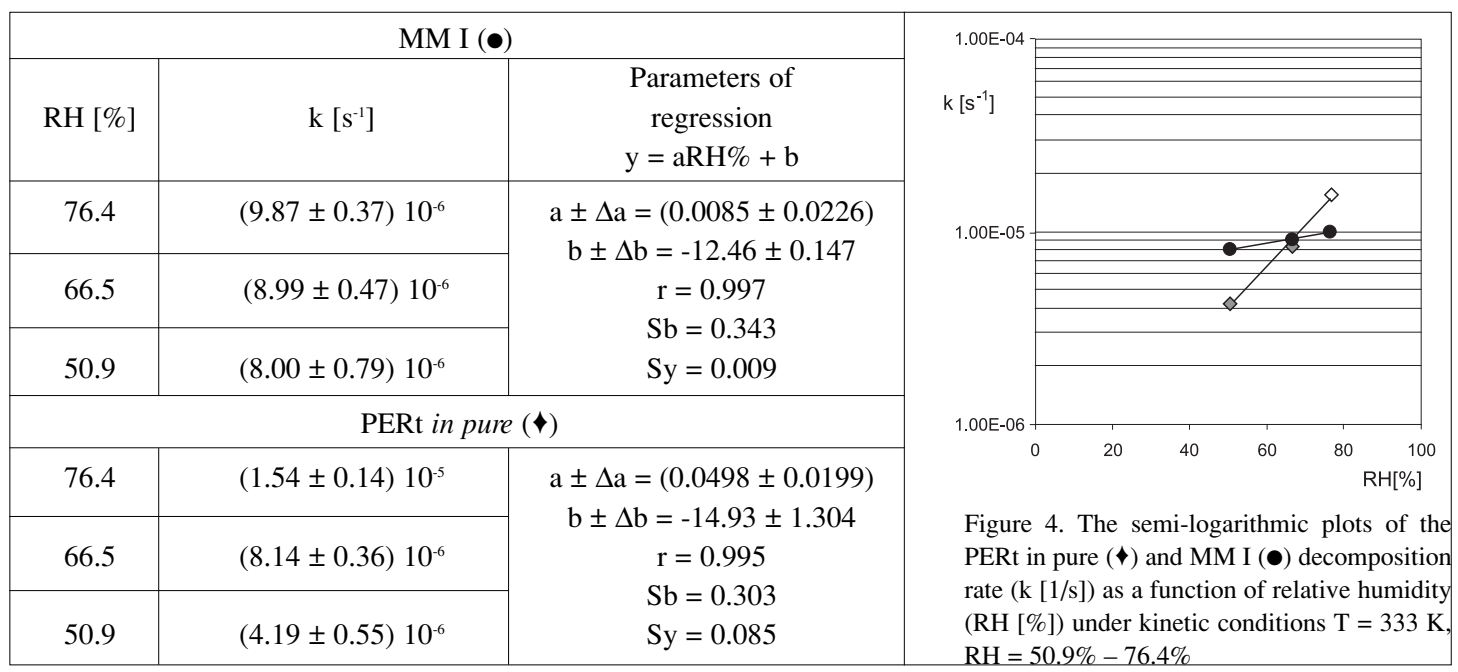


Table 3. The calculated decomposition rate constans of PERa in pure and PERa in model mixture with HTH (MM II); T = 333 K.

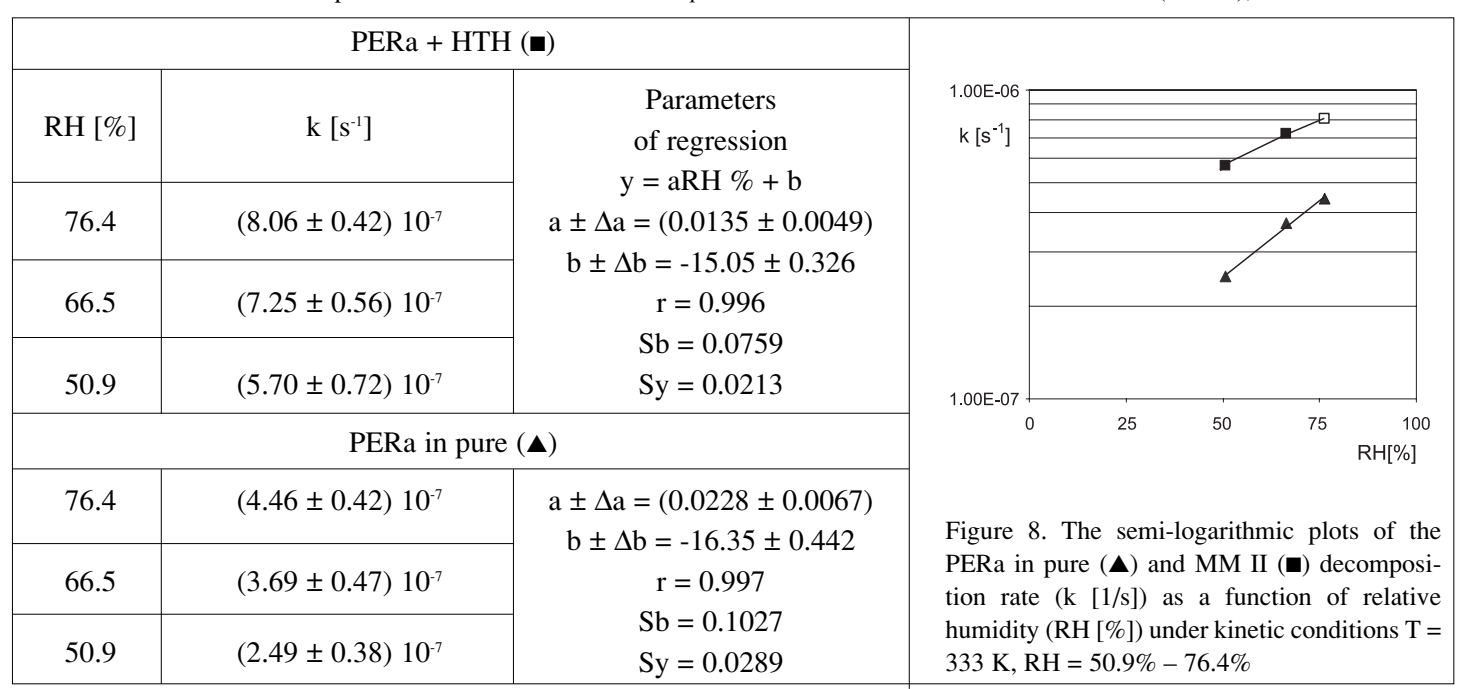
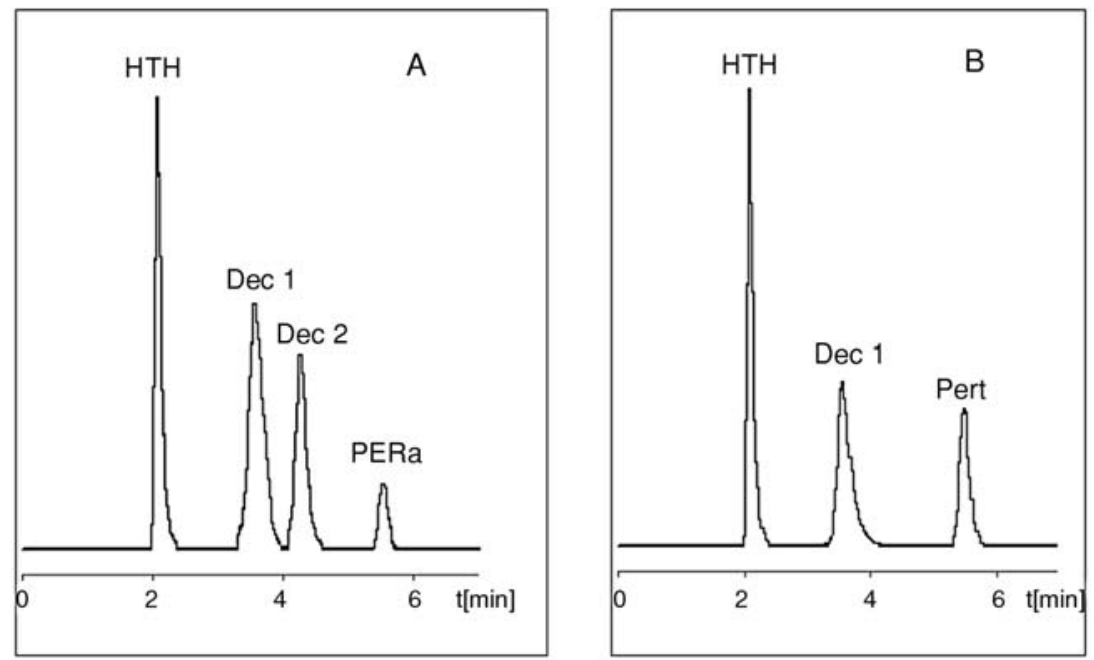

Figure 2. A -RP-HPLC chromatogram of PERa, HTH and products of PERa decomposition (Dec 1 and Dec 2 ) stored at RH $=76.4 \%$, $\mathrm{T}=$ $333 \mathrm{~K}$ for $250 \mathrm{~h}$. B - RP-HPLC chromatogram of PERt, HTH and product of PERt decomposition (Dec 1 ) stored at RH = 76.4\%, T $=333$ $\mathrm{K}$ for $120 \mathrm{~h}$

\section{PERt-HTH model mixture (MM I)}

The relative humidity impact on the stability of analyzed MM I was tested. MM I decomposition reaction at decreased relative humidity and temperature takes place according to the model of autocatalytic reaction, the same as decomposition of PERt in pure. To describe the autocatalytic reaction mathematically, the Prout-Tompkins equation was applied. According to the concept of the ProutTompkins equation, the acceleratory period of autocatalytic reaction after transformation gives a linear relationship (Fig. 3). This can be defined by the equation:

$$
\ln \mathrm{c}_{\mathrm{t}} /\left(\mathrm{c}_{0}-\mathrm{c}_{\mathrm{t}}\right)=\mathrm{C}-\mathrm{k} \times \mathrm{t}
$$

where $c_{t}$ stands for a concentration of PERt after $t$ [h] of incubation period, $c_{0}$ stands for $100 \%$ PERt concentration in solid state before the incubation, $\mathrm{C}$ stands for the parameter related to induction period and $\mathrm{k}$ stands for reaction rate constant at specified $\mathrm{RH}$ and $\mathrm{T}=333 \mathrm{~K}$. It is described by means of the proper curves and the corresponding equations in Table 2.

As decomposition of PERt in pure under the same conditions takes place according to the same model of reaction (Fig. 3), it can be concluded, that presence of HTH does not change the model of reaction. 

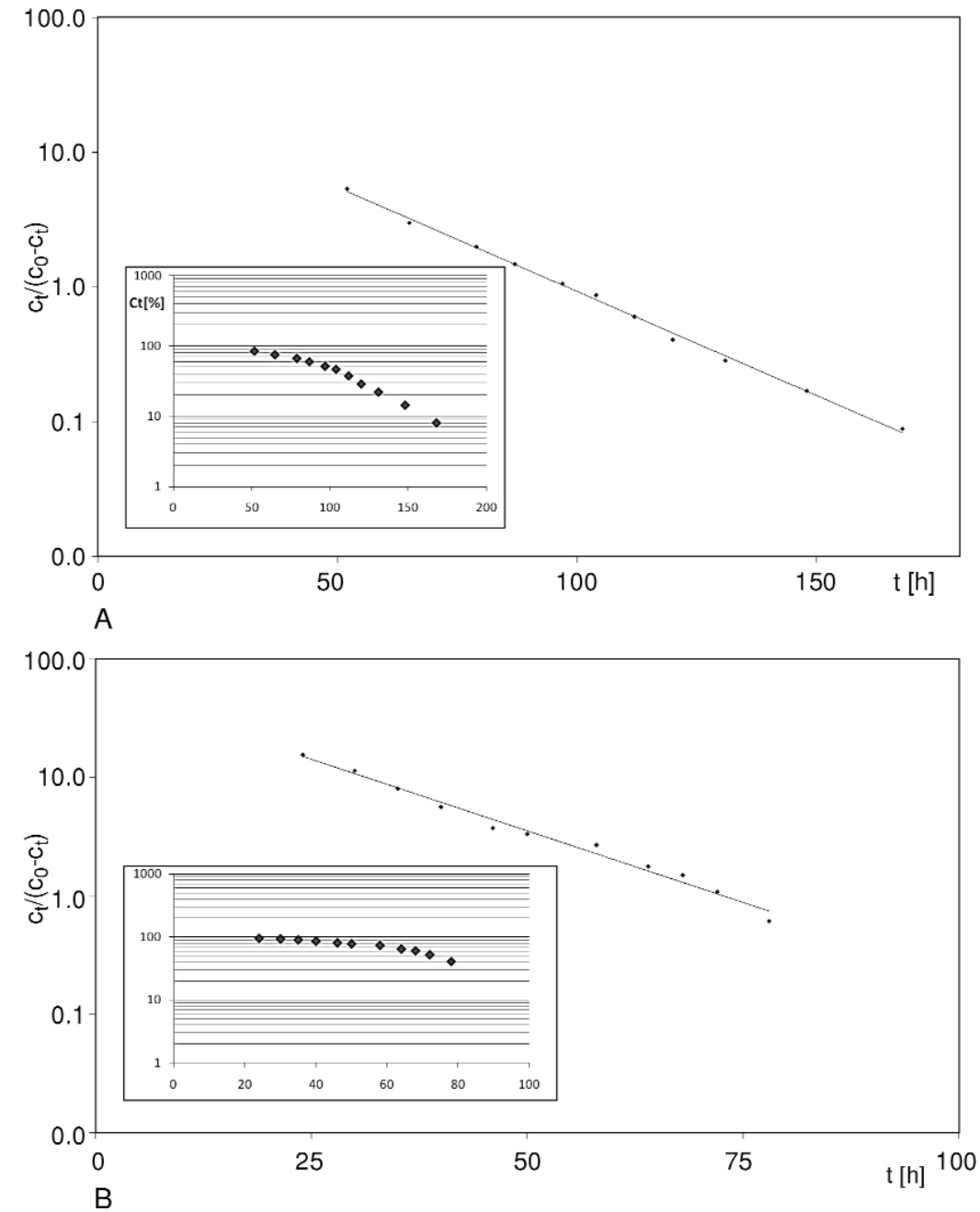

Figure 3. A - Prout-Tompkins transformation of MM I decomposition diagram ( $\mathrm{T}=333 \mathrm{~K}$ and $\mathrm{RH}=76.4 \%)$. Internal graph - Semi-logarithmic plot $c_{t}=f(t)$ of PERt in model mixture with HTH during the stress test - autocatalytic model; B - Prout-Tompkins transformation of PERt in pure decomposition diagram $(\mathrm{T}=333 \mathrm{~K}$ and $\mathrm{RH}=76.4 \%)$. Internal graph - Semi-logarithmic plot $\mathrm{c}_{\mathrm{t}}=\mathrm{f}(\mathrm{t})$ of PERt during the stress test - autocatalytic model
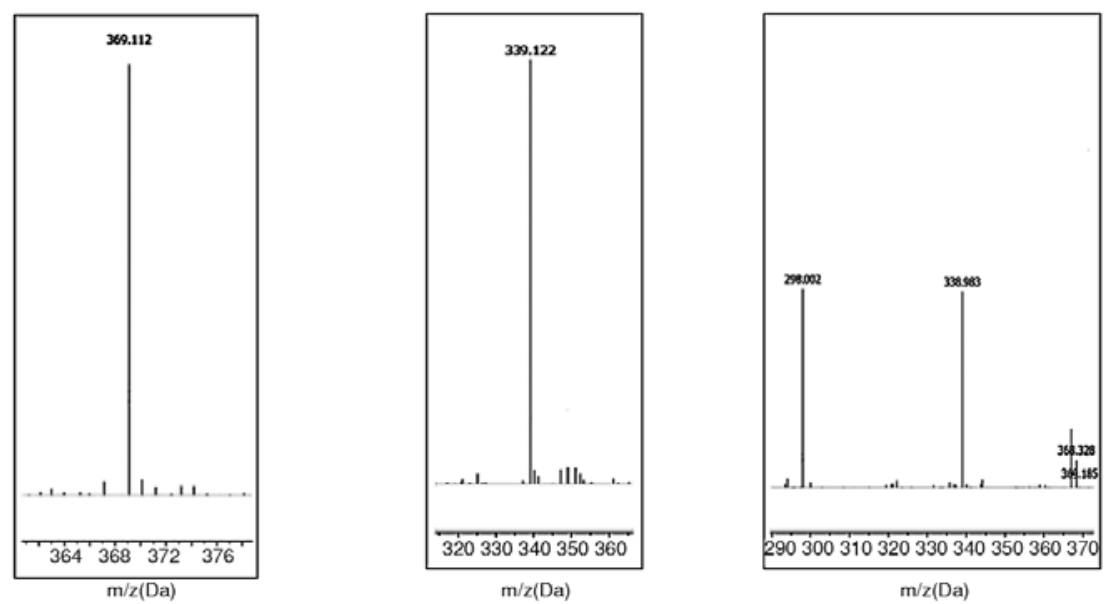

Figure 5. A- $\mathrm{ES}^{+}$mass spectrum of PERt $\mathrm{M}=368 ; \mathrm{ES}^{+} \mathrm{m} / \mathrm{z}=369 ; \mathbf{B}-\mathrm{ES}^{+}$mass spectrum of PERt after incubation process : degradation product : Dec $1-\mathrm{M}=338 ; \mathrm{ES}^{+} \mathrm{m} / \mathrm{z}=339 ; \mathrm{C}-\mathrm{ES}^{+}$mass spectrum of $\mathrm{MM} \mathrm{I}$ after incubation process $: \mathrm{PERt} \mathrm{M}-=368 ; \mathrm{ES}^{+} \mathrm{m} / \mathrm{z}=$ 369 , $\mathrm{HTH}\left(\mathrm{M}=297, \mathrm{ES}^{+} \mathrm{m} / \mathrm{z}=298\right)$, degradation product $:$ Dec $1-\mathrm{M}=338 ; \mathrm{ES}^{+} \mathrm{m} / \mathrm{z}=339$ 


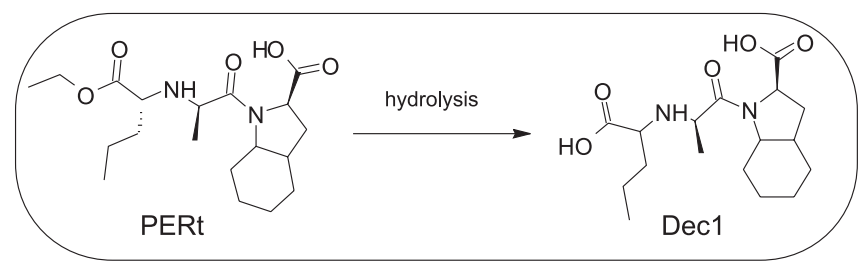

Figure 6. Proposed mechanism of PERt in model mixture with HTH and PERt in pure decomposition, one main decomposition product Dec 1 (hydrolysis product - perindoprilat) can be observed
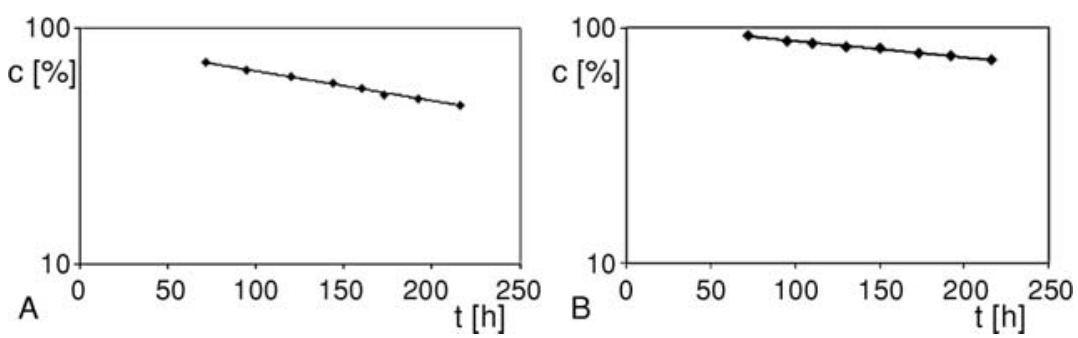

Figure 7. A - Semi-logarithmic plot $c_{t}=f(t)$ of PERa in model mixture with HTH during the stress test - first order kinetic model $(\mathrm{T}=$ $333 \mathrm{~K}$ and $\mathrm{RH}=76.4 \%)$. B - Semi-logarithmic plot $\mathrm{c}_{\mathrm{t}}=\mathrm{f}(\mathrm{t})$ of PERa during the stress test - first order kinetic model $(\mathrm{T}=333 \mathrm{~K}$ and $\mathrm{RH}=76.4 \%)$

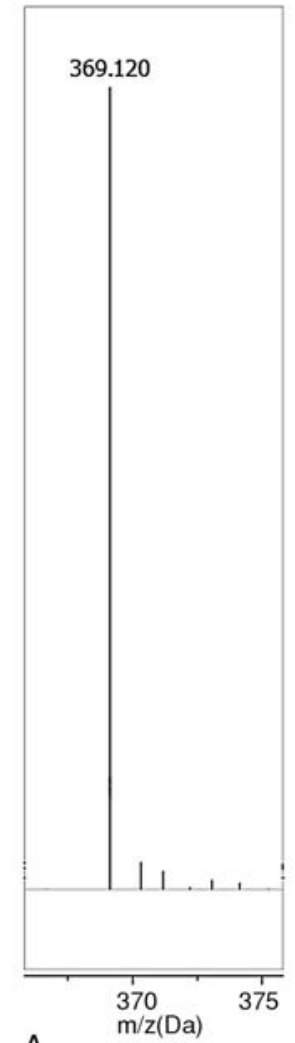

A

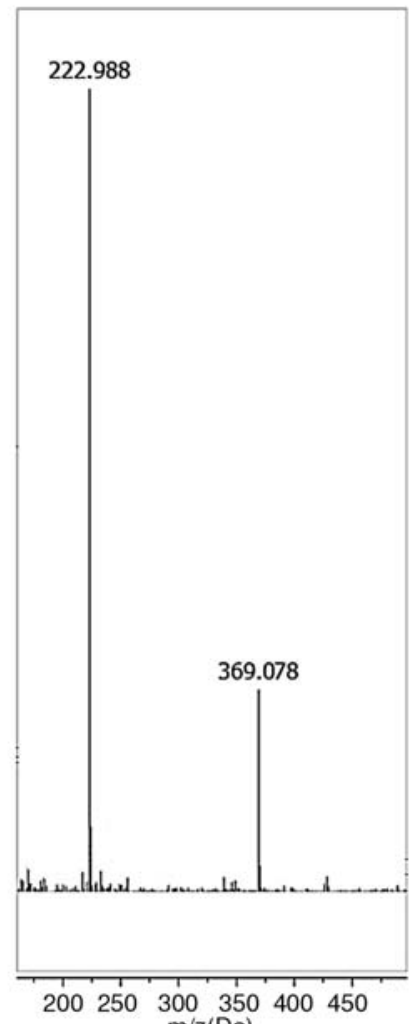

$\mathrm{B}$

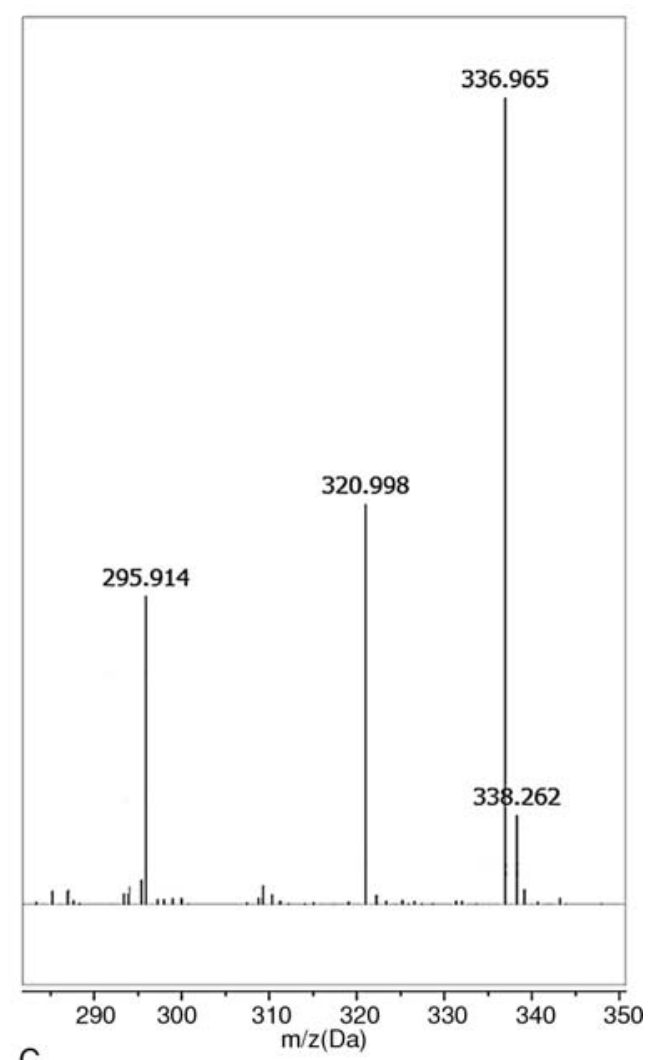

C

Figure 9. A - ES + mass spectrum of PERa M-=368; $\mathrm{ES}^{+} \mathrm{m} / \mathrm{z}=369 ; \mathbf{B}-\mathrm{ES}+$ mass spectrum of PERa after incubation process : PERa $\mathrm{MS}=368, \mathrm{ES}^{+} \mathrm{m} / \mathrm{z}=369$, degradation product: Dec $3-\mathrm{M}-=221 ; \mathrm{ES}^{+} \mathrm{m} / \mathrm{z}=222 ; \mathrm{C}-\mathrm{ES}-$ mass spectrum of $\mathrm{MM}$ II after incubation process : $\mathrm{HTH}\left(\mathrm{M}=297, \mathrm{ES}^{+} \mathrm{m} / \mathrm{z}=298, \mathrm{ES}^{-} \mathrm{m} / \mathrm{z}=296\right)$ degradation products $:$ Dec $1-\mathrm{M}-=338 ; \mathrm{ES}^{+} \mathrm{m} / \mathrm{z}=339 ; \mathrm{ES}^{-} \mathrm{m} / \mathrm{z}=337, \mathrm{Dec}^{-}$ $2-\mathrm{M}=322 ; \mathrm{ES}^{+} \mathrm{m} / \mathrm{z}=323 ; \mathrm{ES}^{-} \mathrm{m} / \mathrm{z}=331$ 
The value of the slope of the regression line $y=$ a $\mathrm{RH} \%+$ b suggests the influence of relative humidity on the drug. Comparison of slope coefficient of PERt in pure and in the presence of HTH suggest that this drug combination in model mixture improve the stability of PERt. The consequence of those substances coexistence is PERt degradation deceleration, which is expressed in kinetic parameters values change: induction time and observed reaction rate constant (Fig. 4). Those differences were statistically significant (Snedecor F-test, $\alpha=0.05$ ). Basing on above, it can be concluded that HTH act as PERt degradation reaction decelerating agent. The chemical reaction between those substances does not occur, while degradation product is the same as in case of PERt in pure substance (Fig. 5). It can be suggested that the product of decomposition process is the product of hydrolysis. The proposed mechanism of PERt degradation is presented in Figure 6, likewise, only one degradation product was observed.

\section{PERa-HTH model mixture (MM II)}

PERa in the MM II decomposition reaction at decreased relative humidity and the temperature takes place according to the different order of reaction - the first order reaction. In comparison with kinetic studies of PERa in pure (Fig. 7) in can be concluded, that HTH does not change the order of decomposition reaction. Comparing the slopes of the curves, it can be seen that the difference is not significant and HTH decreases the process of decompo- sition (Fig. 8). The relative humidity impact on the stability of analyzed drugs and model mixtures was tested. It is described by means of the proper curves and the corresponding equations in Table 3.

The stability study results of PERa in pure and in the presence of HTH comparison suggest that this drug combination in model mixture enhances the stability of PERa. The consequence of those substances coexistence is PERa degradation deceleration, which is expressed in kinetic parameters values change observed reaction rate constant (Table 3). Those differences were statistically significant (FSnedecor test, $\alpha=0.05$ ). The chemical reaction between those substances does not occur, while degradation products are not the same as in the case of PERa in pure (Fig. 9).

It can be suggested that during the decomposition process occur two products: diketopiperazine and product of hydrolysis. The proposed mechanism of PERa in MM II degradation is presented in Figure 10. In comparison, the degradation process of PERa in pure stored in the same condition leads to one main product - diketopiperazine along with breaking off the fragment of the molecule (Fig. 11).

\section{CONCLUSIONS}

For the purpose of the study, an analytical method of determination of PERt and PERa in the presence of HTH has been developed. The proposed RP-HPLC method was validated in compliance with

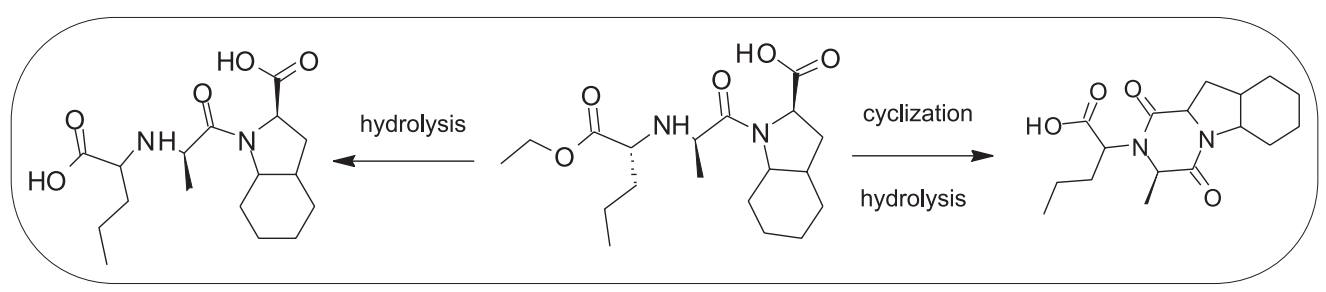

Figure 10. Proposed mechanism of PERa decomposition in the presence of HTH, two main products can be observed (hydrolysis product - perindoprilat - Dec 1 - and hydrolysis and cyclization product - diketopiperazine derivative - Dec 2)

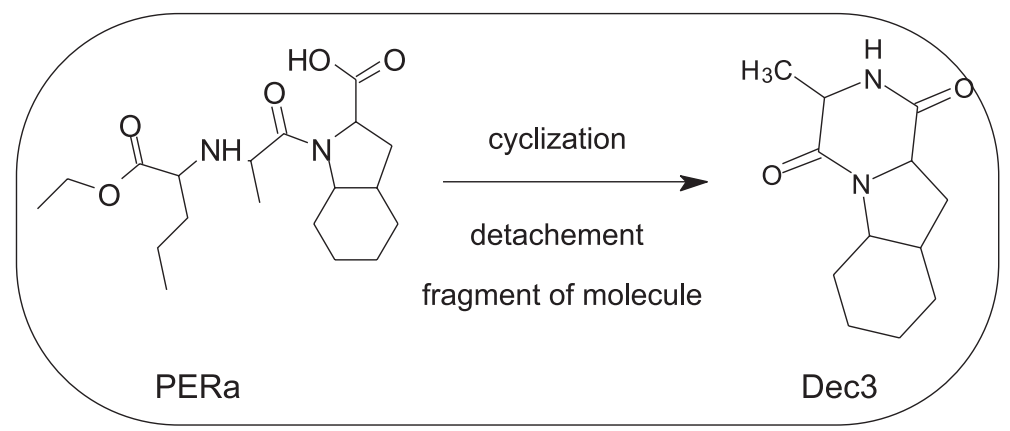

Figure 11. Proposed mechanism of PERa in pure decomposition, one main product can be observed - Dec 3 
validation requirements; it has the required linearity, accuracy, precision, selectivity, LOD and LOQ, and was proved to be capable of separating PERa and PERt, their degradation products and HTH, hence demonstrating its stability - indicating capability. The short analytical run leads to a cost- effective and rapid chromatographic procedure which can be used for routine analysis and for stability studies of samples of PERa or PERt and HTH.

\section{PERt}

Comparison of the stability studies of PERt in pure and in the model mixture with HTH suggests, that HTH does not change the mechanism of decomposition process. What is interesting, stability of PERt greatly increases in the presence of HTH.

\section{PERa}

Comparison of the stability studies of PERa in pure and in the model mixture with HTH suggests, that HTH does not changes the kinetic mechanism of decomposition process, but changes the degradation product. It can be concluded, that HTH affects protectively on PERa molecule and the stability is also influenced by the presence of HTH in model mixture. The interaction occurs in form of the deceleration of PERa degradation reaction: decrease of the observed reaction rate constant.

\section{Impact of $\mathrm{HTH}$}

The authors of these studies unambiguously state that HTH does not have the negative effect on chemical stability of PERt or PERa, moreover HTH works positively on stability of these molecules.

\section{Acknowledgements}

The AJ acknowledge financial support for the project from the PUMS no 502-14-0330541141169. The WS acknowledge financial support for the project from the PUMS no 502-01-0331342708870 .

\section{REFERENCES}

1. Carey R.M., Siragy H.M.: Endocr. Rev. 24, 261 (2003).

2. Sica D.A.: Curr. Hypertens. Rep. 12, 67 (2010).

3. Paszun S., Stanisz B.: J. Med. Sci. 5, 392 (2008).

4. Hurst M., Jarvis B.: Drugs 61, 867 (2001).

5. WHO World Health Statistics Report, 2013.

6. Cowart J.B., Taylor A.A.: Curr. Hypertens. Rep.14, 324 (2012).
7. Wzgarda A., Kleszcz R., Prokop M., Regulska R., Regulski M. et al.: Eur. J. Pharmacol. 797, 9 (2017).

8. Ghiadoni L.: Expert Opin. Pharmacother. 12, 1633 (2011).

9. Paszun S., Stanisz B., Gradowska A.: Acta Pol. Pharm. 70, 1079 (2013).

10. Yoshioka S., Stella V.J.: Stability of drugs and dosage forms. Kulwer Academic Publishers, New York 2002.

11. Crichton B.: J. R. Soc. Med. 97, 328 (2004).

12. Risha P.G., Vervaet C., Vergote G., Van Bortel L., Remon J.P.: Eur. J. Clin. Pharmacol. 59, 135 (2003).

13. Wzgarda A., Dettlaff K., Rostalska M., Pabian E., Regulska K., Stanisz B.J.: Iran J. Pharm. Res. 16, 1007 (2017).

14. Regulska K., Regulski M., Paszun S.K., Wzgarda A., Niemier B. et al.: Arab. J. Chem. 10, 378 (2017).

15. Regulski M., Regulska K., Stanisz B.J., Murias M., Gieremek P. et al.: Curr. Pharm. Des. 21, 1764 (2015).

16. Stanisz B.: Acta Pol. Pharm. 60, 443 (2003).

17. Stanisz B.: Acta Pol. Pharm. 61, 91 (2004).

18. Stanisz B.: React. Kinet. Mech. Cat. 85, 145 (2005).

19. Regulska K., Stanisz B., Lisiecki P.: AAPS PharmSciTech. 14, 1199 (2013).

20. Paszun S., Stanisz B.: React. Kinet. Mech. Cat. 109, 285 (2013).

21. Stanisz B.: J. Liq. Chromatogr. Relat. Technol. 27, 3103 (2004)

22. Stanisz B.: J. Pharm. Biomed. Anal. 31, 375 (2003).

23. International Conference on Harmonization Guidelines, Technical requirements for registration of pharmaceuticals for human use. 62, 27115 (1997)

24. International Conference on Harmonization Guidelines, Technical requirements for registration of pharmaceuticals for human us. Validation of Analytical Procedures. 60, 11260 (1995).

25. Gumieniczek A., Hopkała H.: Chem. Anal. 43, 951 (1998).

26. Erk N.J.: Pharm. Biomed. Anal. 26, 43 (2001).

27. Rani P.A., Sekaran B.C.: Int. J. Pharm. Tech. Res. 1, 575 (2009).

28. Khomushku G.M., Zhloba A.A., Puchnin V.S., Arkhipova M.V., Moiseeva S.M.: Pharm. Chem. J. 44, 619 (2011).

Received: 22.11.2017 